Vivian Strotmann*

\title{
Die Bibliographische Datenbank zur Geschichtsschreibung im Osmanischen Europa (15. bis 18. Jh.)
}

\author{
Ein kooperatives Projekt zur Präsentation von Forschungsdaten
}

https://doi.org/10.1515/bfp-2019-2017

Zusammenfassung: Das DFG-Projekt „Bibliographische Datenbank zur Geschichtsschreibung im Osmanischen Europa (15. bis 18. Jh.)“ (HOE) wurde zwischen 2015 und 2018 kooperativ vom Lehrstuhl für die Geschichte des Osmanischen Reiches und der Türkei (ORT) und der Universitätsbibliothek (UB) der Ruhr-Universität Bochum durchgeführt. Der vorliegende Artikel gibt einen Überblick über die dem Systemdesign zugrundeliegenden konzeptionellen und technischen Überlegungen und beschreibt die Rolle der Universitätsbibliothek in einer Kooperation mit mehreren wissenschaftlichen Partnern. ${ }^{1}$

Schlüsselwörter: Historiografie; Osmanisches Europa; bibliografische Metadaten

The Bibliographical Database for the Historiography of Ottoman Europe (15th to 18th Century)

A cooperative project for the presentation of research data

Abstract: The DFG-funded project "Bibliographical Database for Historiography of Ottoman Europe $\left(15^{\text {th }}-18^{\text {th }}\right.$ Century)" (HOE) was conducted jointly by the Chair for the History of the Ottoman Empire and Modern Turkey (ORT) and the University Library (UB) of Ruhr University Bochum between 2015 and 2018. The article at hand provides an overview of the conceptual and technological considerations that inform the system and describes the positioning of the UB in cooperation with several researching partners.

Keywords: Historiography, Ottoman Europe; bibliographical metadata

1 Teil 1.1 des Textes und die korrespondierenden Internetseiteninhalte beruhen auf Ausführungen von Ovidiu-Victor Olar, Mitglied des wissenschaftlichen Projektteilteams.

*Kontaktperson: Vivian Strotmann, Vivian.Strotmann@rub.de

\section{Projektgenese und -Entwicklung}

\subsection{Wissenschaftlicher Impuls}

Der wissenschaftliche Impuls für das Projekt war die Tatsache, dass die Historiografie des Osmanischen Europa, ${ }^{2}$ insbesondere in ihrer Ausformung in balkanischen Quellen des 15. bis 18. Jahrhunderts, die Aufmerksamkeit von Forschern bisher in eher geringem Maß auf sich gezogen hat.

Um diese Lücke zu füllen wurde 2014 ein DFG-Projekt beantragt, ${ }^{3}$ dessen Ziel die gemeinschaftliche wissenschaftliche und bibliothekarische Erschließung und Präsentation der bisher wenig erforschten Quellen und der ihnen zugehörigen Sekundärmaterialien war.

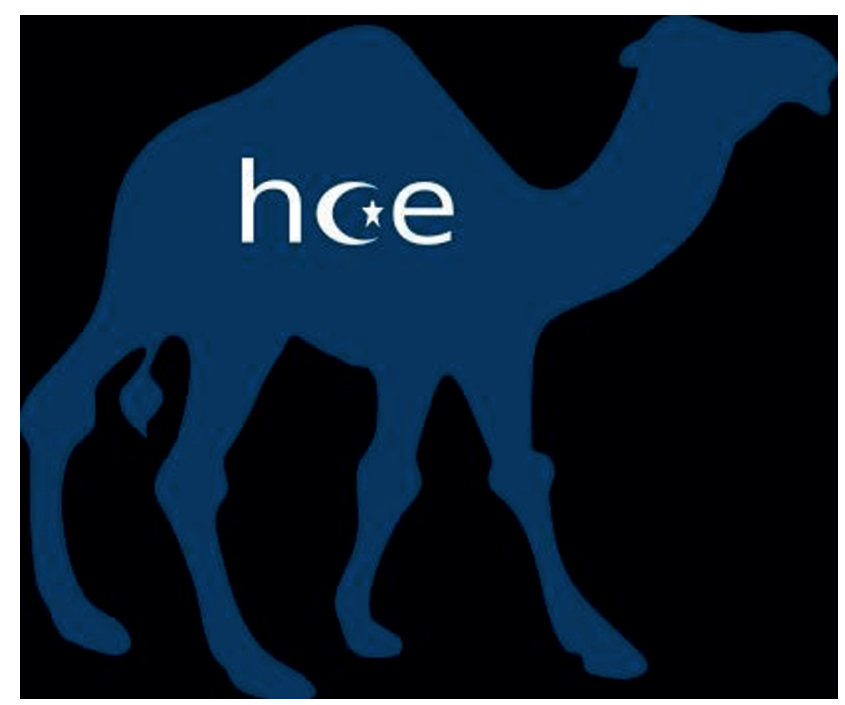

Abb. 1: Projektlogo 4

Hierbei sollten umfangreiche vor-Ort-Recherchen in Bibliotheken, Archiven und Sammlungen weltweit seitens

2 Für eine erste Definition dieses Bergriffes siehe http://www.ub.rub. de/oh/concepts.html.

3 Siehe auch http://gepris.dfg.de/gepris/projekt/249662419.

4 HOE-Projektlogo von André Hagenbruch. 
der Projektmitarbeiter des Lehrstuhls ORT durch infrastrukturelle Unterstützung, konzeptionelle Kooperation und bibliotheksspezifische Datenanreicherung in der UB ergänzt werden. Nach mehreren personellen Änderungen, von denen der unerwartete Tod des leitenden Entwicklers und Designer des Projektlogos ${ }^{5}$ André Hagenbruch, die einschneidenste war, umfasst das zu diesem Zweck versammelte Team folgende Mitglieder:

- Prof. Dr. Markus Koller (Projektleiter), Inhaber des Lehrstuhls für die Geschichte des Osmanischen Reiches und der Türkei der Ruhr-Universität Bochum (ORT)

- Dr. Erdmute Lapp (Projektleiterin), Direktorin der Universitätsbibliothek Bochum (UB)

- Dr. Vivian Strotmann (Metadatenspezialistin \& Onlinerecherche), Wissenschaftliche Mitarbeiterin der UB und des Instituts für Soziale Bewegungen Bochum

- Dr. Ovidiu Olar (Vor-Ort-Recherche \& Geschichtsspezialist), Mitarbeiter des „N. Iorga“ Instituts Bucharest und Affiliated Scholar des Lehrstuhls ORT sowie des Zentrums für Mittelemeerstudien Bochum.

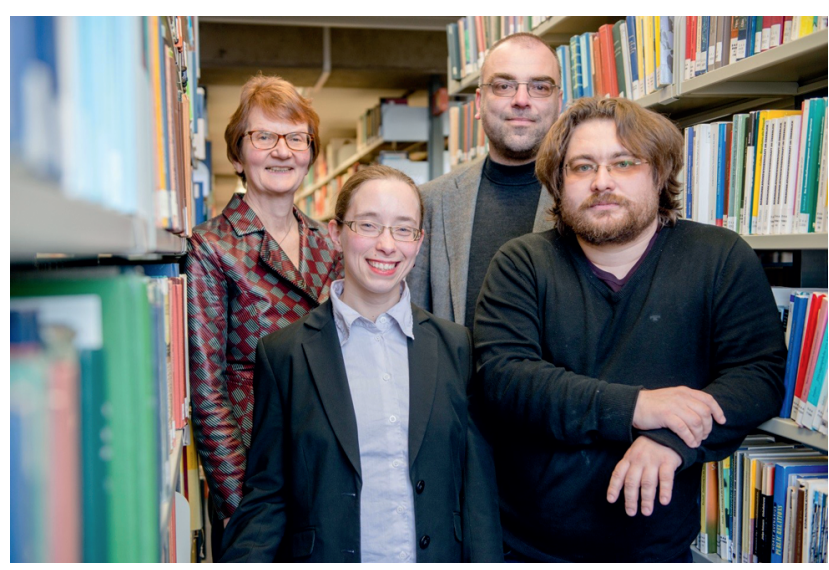

Abb. 2: Teamfoto $\odot$ RUB, Marquard

\subsection{Zentrale Meilensteine und Entscheidungen}

Wichtige Aktivitäten des Projektes über den Zeitverlauf hinweg sind detailliert auf den entsprechenden Informationsseiten $^{6}$ des Projektes dokumentiert. Im Wesentlichen besteht die Onlinepräsenz des Projektes aus zwei verschiedenen Seiten:

5 Siehe hierzu http://www.ub.rub.de/oh/history.html.

6 Siehe http://www.ub.rub.de/oh/activities.html.
1. der Informationsseite zum Projekt unter http://www.u b.rub.de/oh/index.html und

2. der Systemseite selbst unter https://hoe.ub.rub.de ${ }^{7}$

Als Host und Betreuer dieser Seiten wurde die Universitätsbibliothek als spezialisierter Dienstleister ihrer wissenschaftlichen Partner tätig. Wie sich im Folgenden im $\mathrm{Zu}-$ sammenhang mit dem System selbst zeigen wird, differenzierte und weitete sich die Rolle der Bibliothek im Verlauf des Projektes aus, sodass sie auf konzeptioneller und inhaltlicher Augenhöhe mit den Projektmitgliedern des Lehrstuhls ORT agierte.

Zwei Erkenntnisse haben die Gestalt und den Verlauf des Projektes maßgeblich geprägt. Erstere betrifft die Rolle und Position der Universitätsbibliothek im Verhältnis zu ihren forschenden Partnern im Gesamtkontext des Projektes (siehe unten). Die zweite betrifft eine Entscheidung und zugleich einen der wichtigsten Meilensteine des Projektes, nämlich die Erkenntnis, dass bestehende Programme, wie z. B. Citavi, das zu nutzen zunächst angedacht und getestet worden war, den Bedürfnissen des Projektes nicht gerecht werden konnten.

\section{Die Bibliografische Sammlung}

\subsection{Struktur und Funktionalitäten}

Somit trifft auf das HOE-Projekt Patrick Sahles Beobachtung zu, dass „technische Lösungen letztlich immer Ausdruck methodischer Ansätze sind“. ${ }^{8}$ Maßgeblich für die Entscheidung, nicht auf bereits existierende Systeme zurückzugreifen, sondern ein eigenes System zu entwickeln, war die Tatsache, dass eine differenzierte Kategorisierung der Einträge auf unterschiedlichen Ebenen erforderlich war, wie sie in gängigen Systemen nicht oder nur unzureichend abgebildet werden kann.

Diese differenzierte Klassifizierung von Einträgen diente zum einen der analytischen Aufarbeitung der Recherchefunde der Mitarbeiter des Lehrstuhls ORT im Vorfeld der Verarbeitung, Validierung und Komplementierung seitens der Universitätsbibliothek. Zum anderen fügte sie der Bandbreite an Filterfacetten entscheidende Elemente

7 Aus dem Projektverlauf ergaben sich als Nebenprodukte eine knappe Seite mit Informationen zum Projekt, unter http://www.ub.ruhr-u ni-bochum.de/projekte/osmanisches_reich.html, ebenso wie eine unter OMP in der UB gehostete Working Paper Series auf https://omp.u b.rub.de/index.php/HOE/index. Letztere wird vom Team Publikationsdienste der UB betreut https://www.ruhr-uni-bochum.de/oa 8 Vgl. Sahle (2017) 151. 
hinzu, die spezifische Suchanfragen und somit speziellen Zugang zu Teilen des erfassten Corpus eröffnen. Die übergeordnete Eintragsqualifizierung als „key publication yes/no“ und als „source class - primary/secondary“, auch im Counter der Startseite genutzt, erlaubt dem Nutzer somit einen unzweideutigen und zugleich intuitiven Zugriff auf die analytischen Grundannahmen der recherchierend/wissenschaftlich tätigen ORT-Mitarbeiter.

Die Filter und Verlinkungen des Systems sind darüber hinaus strukturelle Manifestation des Umstandes, dass die Methodologie der Quellenerschließung, -Kontextualisierung und -Präsentation neben einer hierarchischen Gliederung in über- und untergeordnete Werke bzw. Werkteile auch Möglichkeiten zur inhaltlich-assoziativen Verknüpfung notwendig machte. Diese inhaltlich-assoziativen Bezüge von Werken/Datensätzen untereinander gliedern sich in die Kategorien „primary literature“/,secondary literature“, „reference“ und „other“. Ein entscheidender Vorteil der projektinternen Systementwicklung und zugleich ein entscheidender Faktor für die Nachhaltigkeit des Unternehmens ist hierbei das hohe Maß an Adaptivität, das es den Programmierern erlaubt, das HOE-System an Bedarfe neu erschlossener Quellentypen anzugleichen.

\subsection{Nachhaltigkeit und Nachnutzung}

Zugleich stellt die Anpassungsfähigkeit, die mit der Entwicklung eines eigenen Systems verbunden ist, auch einen der entscheidenden Vorteile des Systems mit Blick auf die Langzeitverfügbar/Migrationsfähigkeit der Daten dar. Deren Sicherung zählt gemäß der im Kontext von ,Digital Humanities'-Projekten weit verbreiteten Rollenverteilung zu den Kernkompetenzen und -Verantwortungen der Universitätsbibliothek. Die Strukturen des Systems wurden so angelegt, dass ein Export der Daten auf Knopfdruck ermöglicht, die Inhalte in ein einfaches Format zu überführen, das der Sicherung ebenso dienen kann, wie der Migration in eine andere Umgebung. Mit Blick auf die projektexterne Nachnutzbarkeit der Daten ist dies für DH-Projekte selbstverständlich zu ermöglichen. Die Metadatensammlung exklusive ins System geladener externer (Bild- oder PDF)Inhalte oder verlinkter Inhalte ${ }^{9}$ - wurde darüber hinaus im Sinne des Open Access unter eine liberale CC-Lizenz gestellt. ${ }^{10}$

Zur kontinuierlichen Sicherung der Speicherbedarfe des Systems und der in ihm verzeichneten Datensätze

9 Siehe http://www.ub.rub.de/oh/disclaimer.

10 Siehe https://creativecommons.org/licenses/by-sa/4.0. stehen die Projektmitarbeiter darüber hinaus im Austausch mit dem Projekt Forschungsdatenmanagement der Ruhr-Universität Bochum. ${ }^{11}$

Eine Dokumentation der Strukturen wurde von den technischen Spezialisten der UB Bochum erstellt. ${ }^{12}$ Derzeit wird darüber hinaus an einer umfangreichen Beschreibung des Systems gearbeitet, die als User Guide für externe Nutzer zur Verfügung stehenden wird, die das moderierte Einreichungstool für neue Inhalte nutzen möchten. Diese Beschreibung wird nach Fertigstellung als HOE Working Paper frei verfügbar sein. ${ }^{13}$

\subsection{Die Bibliothek und das Verständnis von ,Digital Humanities‘}

Die Rolle der Universitätsbibliothek im Kontext des Projektes sollte sich nicht auf die oben beschriebene Daten bewahrende und unterstützende Funktion beschränken. Dies war ein weiterer Grund für die gemeinsame Konzeption und Implementierung eines projekteigenen Systems.

Neben der Abbildung analytischer Kategorien von Quellengruppen und inhaltlich-assoziativer Bezüge zwischen einzelnen Datensätzen, welche durch die Forscher des Lehrstuhls ORT definiert wurden, sollte der DigitalHumanities-Facette des Projektes vor allem durch eine Vielzahl sorgsam konzeptualisierter Filter, Visualisierung geografischer Zusammenhänge mittels in Karten übertragener Geodaten, Komplementierung der Verschlagwortung durch Links zu DB-Pedia sowie umfangreiche Verlinkung von Datensätzen mit systemexternen (OA)Ressourcen aus der immer weiter wachsenden Bandbreite von born-digital-Projekten und digitalisierten Materialien, die inzwischen online zu finden sind, Rechnung getragen werden.

Neben Verknüpfungen mit im Volltext verfügbaren Inhalten beinhaltete diese Herangehensweise an die Aufwertung der wissenschaftlich aufbereiteten, bibliografisch in der Bibliothek geprüften und komplementierten Datensätze vor allem die Einbindung/Verlinkung unterschiedlicher bibliotheksspezifischer Kennungen und Identifier für Werke ebenso wie für die an ihrer Entstehung beteiligten Personen.

11 Weitere Informationen hierzu unter https://www.ruhr-uni-bo chum.de/researchdata.

12 Einen GitHub-Eintrag von 2016, von André Hagenbruch erstellt, findet sich darüber hinaus unter https://github.com/ubbochum/hoe. 13 Publikationen werden laufend auf der entsprechenden Informationsseite des Projektes angekündigt: http://www.ub.rub.de/oh/activ ities.html bzw. http://www.ub.rub.de/oh/publications.html. 

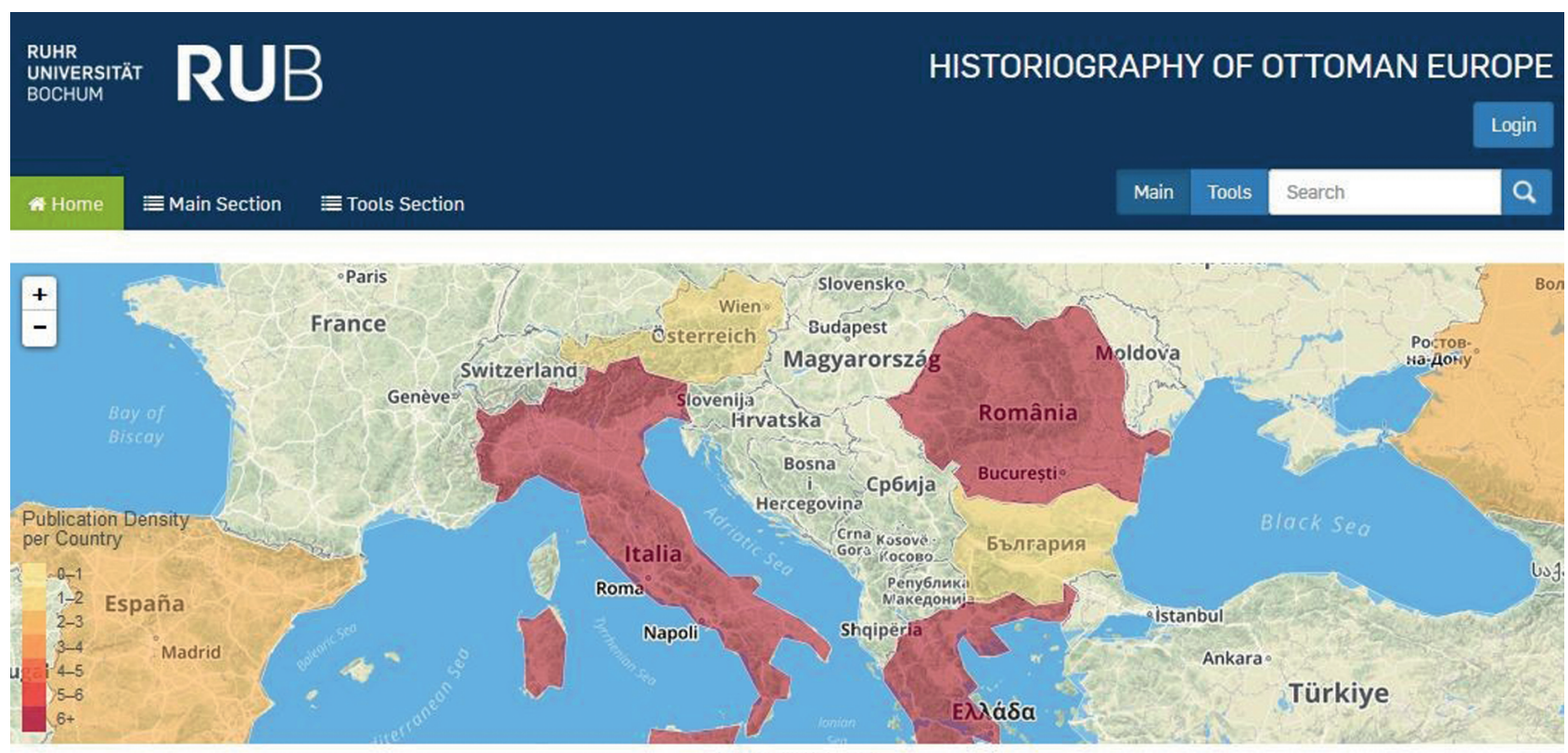

Number of Publications

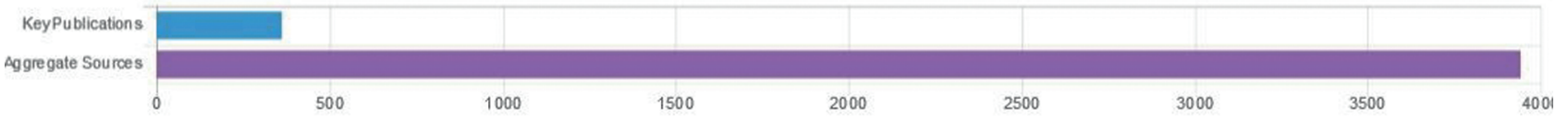

Welcome to the "Historiography of Early Modern Ottoman Europe" (HOE) Database!

The information at your disposal on these pages results from a DFG-funded project based on synergy of expertise between the Chair for the History of the Ottoman Empire and Modern Turkey of Ruhr-Universität Bochum and the University Library Bochum. More information on the history, outlook, and outreach of HOE is available here.

\section{What is HOE?}

$\mathrm{HOE}$ is a collection of meta-data focusing on historiographical writing of Early Modern Ottoman Europe
What does it do?

HOE provides information on the author(s), title(s), contents, manuscript(s) and edition(s) of the main

\section{How to use it?}

You can switch between the Main Section and the Tool Section at will Different facets and filters will help you

\section{What to keep in mind?}

In accordance with the maxims of open access, we are making our collection of meta-data freely accessible under $\mathrm{CC}$ -

Abb. 3: Startseite HOE-Metadatensammlung ${ }^{14}$

Diese von der Bibliothek betreuten Verlinkungen stellen einen integralen Bestandteil der spezifischen Inhaltspräsentation und -Erschließung dar und eröffneten der UB Bochum somit auch Handlungsfelder jenseits der klassischen Dienstleistung und Unterstützung. Das differenzierte und mit unterschiedlichen weiterleitenden, beschreibenden, kontextualisierenden oder Volltexte bereitstellenden Verknüpfungen angereicherte Gesamtbild der im System erfassten Texte war neben der wissenschaftlichen Erschließung der unbekannten Quellen maßgebliches Ziel des Projektes. Deshalb wurden die Strukturen, Inhalte, Bedarfe und Abläufe des Systems synergetisch und gleichberechtigt von beiden Projektparteien, dem Lehrstuhl ORT und der UB Bochum, konzipiert und operativ in der UB implementiert.

14 https://hoe.ub.rub.de.
Neben der Entwicklung projektspezifischer Systemstrukturen wird der Begriff der Digital Humanities im Kontext der HOE-Datenbank somit einerseits primär im Sinne der technisch verbesserten Präsentation von Forschungsdaten verstanden, die dem Betrachter eine in weiterführende Kontexte eingebettete erste Wahrnehmung erlaubt. Dies kann nicht nur die Wahrnehmung einzelner Facetten schärfen, die die quellenerschließenden Wissenschaftler als zentral erachteten und deshalb strukturell im HOESystem abbilden ließen.

Es kann auch als Service für Nutzer der Seite begriffen werden, für die sich ein Teil des Rechercheaufwandes erübrigt und denen das HOE-System - ähnlich wie klassische Bibliothekskataloge - somit als Rechercheinstrument dienen kann. Darüber hinaus erlaubt die Kombination aus internen und externen Links und umfangreichen Filterfacetten auch neue Herangehensweisen an das Corpus der erfassten Werke und kann dadurch neue Forschungsfragen offenlegen. Durch die systemspezifische Einbettung 
und Interkonnektivität der Metadatensätze, ebenso wie durch die komplementierenden Daten und externen Verbindungen entsteht ein Mehrwert, der über die reine bibliografische Angabe hinausreicht. Er vergrößert die Nutzbarkeit und Aussagekraft der Datensätze des HOE-Systems erheblich. Dadurch ist das System nicht nur ein nutzerfreundliches Recherchewerkzeug, sondern auch selbst ein potenzieller Forschungsgegenstand, bzw. eine Erkenntnisquelle.

Es handelt sich bei der „Bibliographischen Datenbank zur Geschichtsschreibung im Osmanischen Europa (15. bis 18. Jh.)“ somit einerseits um ein spezifisches System zur Präsentation von Forschungsdaten (das ggf. im Kontext vieler anderer Projekte von Nutzen sein kann). Zum anderen handelt es sich um ein Projekt im Sinne der Digital Humanities, das seine Inhalte hinsichtlich Nutzerfreundlichkeit und Forschungsrelevanz mittels der systemspezifischen Strukturen aufgewertet hat. Auf die in diesem Kontext relevanten Charakteristika des Systems kann im gegebenen Rahmen nicht im Einzelnen eingegangen werden, sie werden jedoch in zukünftigen Publikationen näher beschrieben werden.

\section{Projektkonsolidierung und Perspektiven}

Neben den oben erwähnten Aspekten der Sicherung der Langzeitverfügbarkeit, Migrationsfähigkeit und Nachnutzbarkeit der Daten durch die UB Bochum fußt die Konsolidierung des Projektes für die Zeit nach Ablauf der Drittmittelförderung (Mitte, bzw. Ende 2018) insbesondere auf den Vorkehrungen, die im Interesse des weiteren Anwachsens des Quellenkorpus getroffenen wurden. Während die Mitarbeiter der UB weiterhin die technische und bibliografische Qualitätssicherung des Projektes tragen werden, wurde eine Möglichkeit zur Interaktion mit dem System über das erwähnte moderierte Einreichungsmodul für alle interessierten Besucher und Nutzer der Seite geschaffen.

Im Sinne der projektdefinierenden kooperativen Ausrichtung zwischen beiden Projektparteien, Lehrstuhl ORT und UB Bochum, wird die wissenschaftliche Qualitätssicherung von Forschern getragen werden. Um die kontinuierliche Weiterentwicklung des Corpus mit hochqualitativen Daten $\mathrm{zu}$ sichern, wurde ein Advisory Board ${ }^{15}$ zusammengestellt, dessen größtenteils forschende Mitglieder sich gegen Ende 2018 koordiniert vom Lehrstuhl ORT und der UB konstituiert haben, um zukünftig in gemeinschaftlicher Absprache eingereichte oder neu ermittelte Datensätze auf ihre Relevanz zu prüfen.

Neben diesen Schritten zur Sicherung des langfristigen technischen und inhaltlichen Fortbestandendes der etablierten Projektinfrastrukturen stellt die Einbettung in eine weiter gefasste fachspezifische Infrastruktur eine der vielversprechendsten Perspektiven für das HOE-Projekt dar. Erste konkrete Überlegungen in dieser Hinsicht werden derzeit unternommen und werden nach der Implementierung auf den genannten Internetseiten kommuniziert werden.

Sollten sie Anregungen in dieser Hinsicht oder andere Rückmeldungen haben, bzw. sich als Forscher oder Mitglied einer Bibliothek oder eines Archives dem Advisory Board anschließen wollen, wenden sie sich bitte an Ottom anhistoriography@rub.de.

\section{Literaturverzeichnis}

Sahle, Patrick (2017): Archiv und Edition: Standards und Best Practice. In: Digitales Edieren im 21. Jahrhundert (Veröffentlichungen des Landesarchivs Nordrhein-Westfalen, 67), hg. v. Martin Schlemmer, 143-64, Essen: Klartext.

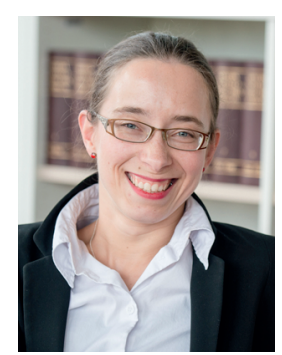

Vivian Strotmann

Universitätsbibliothek Bochum

Raum 6/09

Universitätsstraße 150

44801 Bochum

Vivian.Strotmann@rub.de

Copyright @RUB, Marquard 